\title{
Study on Health Education and Health Knowledge of Female College Students
}

\author{
Haina Cui, Tingting Xie
}

College of Nursing, Binzhou Polytechnic, Shandong City, Binzhou Province, the Yellow River City, twelve Road, No. 919, 256603,China

Keywords: Primary dysmenorrheal, Health education, Female Students

\begin{abstract}
Primary dysmenorrhea seriously affects the personal life and learning, through the system of health education, can alleviate the dysmenorrhea, so as to improve the quality of life and learning effect.
\end{abstract}

\section{Introduction}

Dysmenorrhea is one of the most common gynecologic symptoms, refers to a line through before and after the menstrual period appear abdominal pain, falling inflation and with backache or other discomfort, degree badly influences the quality of work and life [1]. Dysmenorrhea can be divided into primary dysmenorrhea and secondary dysmenorrhea, dysmenorrhea (PD) also known as functional dysmenorrhea, more common in adolescent females, unmarried and married nulliparous, since after menstruation began, for the first day of the most painful drama, continuous 2-3 days to ease, a don't serious can shock syncope. Higher vocational nursing students age multi ranged from 19 to 23 years old, is a primary dysmenorrhea in high-risk population, the daily study and life is often affected by the serious influence, some even for menstrual cramps produced psychological fear. Over the past two years, the author found that the focus on the implementation of health education for girls with primary dysmenorrhea, can reduce the pain of nursing students, improve the quality of life and health.

\section{Content of health education}

Publicity related knowledge, so that nursing students understand menstruation and the correct treatment of primary dysmenorrheal. Some girls because of menstrual period, menstrual blood out of the person's daily activities and even cause embarrassing phenomenon, so on menstrual cramps disgust; also some girls because of primary dysmenorrhea menstruation full of fear, so often the menstrual cramps called "recently I want bad thing", "bad time", "suffer the day", for menstrual cramps don't have the right attitude. Therefore, it is necessary to explain to nursing students knowledge about menstruation, including menstruation appears the reason, significance, menstrual discomfort.

The onset of menstruation is the result of the interaction between the reproductive hormones in hypothalamus, pituitary and ovary, menstruation is symbol of the onset of puberty in female, is one of the sign of the maturation of the female reproductive organs, is essential in the life of a woman "friends." In the menstrual period, generally no specific symptoms, but some women can lower abdominal or lumbosacral falling discomfort or uterine contraction pain, and accompanied by diarrhea and other gastrointestinal function disorder, also is the emergence of dysmenorrhea. 
Primary dysmenorrhea reason is various, Chinese tend to think of poor blood circulation, qi stagnation and blood stasis is the root cause of dysmenorrhea, "no pain" is Chinese the most fundamental point of view. Western medicine believes that the main and menstrual pelvic congestion and excessive activation of the prostaglandin, but improper diet, emotional irritability, cold and dampness can increase the dysmenorrhea. Primary dysmenorrhea can be prevented, alleviated and treated.

\section{Strengthen menstrual health care}

Nurse college students, although the medical students, but menstruation self-care awareness is very weak, some during the menstrual period have the habit of eating cold, some of the girls to slim in menstrual wear thin clothes, some girls even during the menstrual period in school organization's relay race, also some because of fear or dysmenorrhea oral drugs in order to postpone menstrual cramps, these bad habits can aggravate dysmenorrhea, so should strengthen menstrual health care education.

Keep good psychological state. Nursing students because dysmenorrhea experience, patients before each menstrual cramps appear anxiety phenomenon, "but also the pain is dead" self suggestion, in front of the menstrual cramps, it is prone to emotional and irritability and other symptoms and improve the feelings of pain, and when the dysmenorrhea and increased anxiety, thus creating a vicious cycle. Therefore shall inform the dysmenorrhea and psychological nursing students, enjoin the menstrual period to maintain emotional stability, relax pain will reduce also suggested that put more focus on interested in things, such as listening to soft music or reading interesting books, in order to dilute the sense of pain by; and encourage nursing students with classmates and friends for emotional communication. Through the above methods to make nursing students to eliminate anxiety and fear of the psychological, maintain a good attitude, the correct treatment of dysmenorrhea, so that it successfully passed the special period.

Proper rest, work and rest. Girls in the classroom generally don't like to leave the desk, and when day of rest and play for a long time to go shopping, sitting and standing will affect the lower limb blood running, caused by abdominal blood not free, increase the pelvic congestion, so in the premenstrual and menstrual should participate in appropriate sports, such as walking, jogging, yoga, etc., which is good for the blood circulation in the legs and pelvis and can enhance the body resistance. Zhang Xingdong [2] in the juvenile sports school survey found that patients with primary dysmenorrhea accounted for only about 3\%, far lower than the less movement of ordinary women, so the movement to alleviate dysmenorrhea female has a important significance. Of course, due to the special physiological characteristics of menstruation, and do not perform strenuous activities, such as high jump, long jump, running, playing football will induce or aggravate the systemic discomfort during menstruation, and even cause dysmenorrhea. Also due to menstrual uterine mouth is ajar state, bacteria easily penetrated the uterine cavity to increase the chance of infection, so the menstrual period is not suitable for swimming. And menstrual period should also pay attention to the rules of daily life, work and rest, to ensure adequate sleep, severe dysmenorrhea nursing students should leave bed rest.

Pay attention to menstrual health. Women in the menstrual period should be and the timely replacement of the use of disinfection of soft cotton sanitary napkins and toilet paper, but also from time to time with warm water to clean the vulva, not tub or bath should take a shower is good; do not wear tights, influence blood backflow, underwear should choose pure cotton and should be changed frequently wash; after defecate formerly wiped backward, lest dirt into the vagina, cause 
reproductive system in the acute and chronic inflammation, resulting in inflammatory dysmenorrhea.

Reasonable arrangement of diet. Nursing students should be advised to arrange a scientific diet, in the menstrual period to pay special attention to diet, eat light, easy to digest food, to maintain smooth stool. Should not eat salty food, the salt and water storage in the leaves increased, especially prone to menstrual edema girls; not suitable for drinking tea, coffee, alcohol, caffeine content is very high in this type of beverage, easy to stimulate the nervous and cardiovascular, resulting in menstrual pain, menstruation and menstrual blood too much discomfort; should not eat cold vegetables fruit and cold drinks, because it will reduce the speed of blood circulation, thereby affecting the uterine contraction and discharge blood, causing blood from adverse, causing dysmenorrhea; should not eat onions, ginger, garlic, pepper and other spicy food, these foods can stimulate uterine smooth muscle and oviduct contraction, thus induce and aggravate dysmenorrhea.

Cold moisture. Support the average age multi in 23 years of age, it is like to dress up in age, some nursing students to beauty even in winter wear very thin, and menstruating women by cold stimulation causes pelvic vascular excessive contraction can aggravate dysmenorrhea. Menstrual period students to resist the cold ability is abate, therefore the guiding students to keep warm, to try to improve the indoor temperature, due to increase the wearing of clothing. Do not drink cold water, wash your face, brush your teeth or washing should use warm water, especially in the cold winter. In the summer, to wash clothes, dry clothes frequently, to wash in cold water to prevent rain, wading.

Provide simple treatment and nursing methods. Primary dysmenorrhea although it is disease of a kind of Department of gynaecology, but not all of the nursing students are required to go to a hospital treatment nor all nursing student need drug treatment. To nursing students to provide some simple care methods to guide nursing students to self commissioning, such as bed rest; pressing Qihai, Guanyuan; massage Hegu, Sanyinjiao; hot compress abdomen median. If no relief dysmenorrhea drinking motherwort, Yueyueshu granule or oral Yuanhuzhitong tablets and other drugs, Bloven. Of course for severe dysmenorrhea nursing students if invalid in a variety of approaches, should urge, persuade, or accompany nursing students go to hospital for examination and treatment as early as possible, to reduce or relieve students of pain, western medicine pays much attention to the "table", intramuscular injection of atropine or 654 - 2 antispasmodic drugs TCM pay attention to "cure" and traditional Chinese medicine decoction has a good therapeutic effect.

\section{Methods of health education}

Health education is through a planned, organized, systematic educational activities, encourage people to consciously adopt useful healthy behavior and way of life, to remove or reduce risk factors that affect the health of, disease prevention, health promotion and improve the quality of life [3]. Health education is not a simple sermon, but a process for students to self experience, self perception, self-improvement. Therefore, to take care of the health of the education form, is to improve the effectiveness of health education Guan Jian.

(1) Set menstrual health education courses. Set the "women's health" public elective courses, to let the nurse system learning menstrual health care knowledge and dysmenorrhea treatment, while utilizing the multimedia devices to play related audio-visual propaganda film.

(2) Teachers of the subtle. High nursing students in school during the study of "obstetrics and gynecology nursing," this course, therefore, teaching activities should be the main way to implement dysmenorrhea health education. Teachers should according to the characteristics of the 
course and teaching should grab the attention of the best combination of theoretical knowledge and dysmenorrhea health education, menstruation and dysmenorrhea related theoretical knowledge to explain to the nursing students, as long as there is skill, consciousness to health education should be infiltrated in the usual spot vocational school, will be able to better achieve "moisten things silently" education effect.

(3) The establishment of a variety of promotional channels, such as the issuance of a brochure, the establishment of a publicity column, recommend the website for students to browse learning, posting publicity pictures, organization and nursing students to watch the science and education, etc.. Through a full range of education, high nursing girls have a more comprehensive understanding of primary dysmenorrhea, enhance the self health care awareness.

(4) The opening of menstrual health education lectures. According to the content of health education, from the school of Obstetrics and gynecology nursing teachers or invited outside of Obstetrics and Gynecology experts, targeted to hold a variety of menstrual health knowledge seminar.

(5) Construction of the college girls health counseling room, open consultation line, for the high nursing students to conduct individual counseling, answer questions related to dysmenorrhea.

\section{The significance of health education}

Science of menstrual health education can reduce and avoid dysmenorrhea through of nurse college students to carry out health education, effectively improve the nursing students of primary dysmenorrhea of understanding and ease dysmenorrhea ability, improve nursing students' negative psychological reaction, establish healthy behaviors, dysmenorrhea can be obtained and the mitigation and treatment, so as to relieve the pain of nursing students, and better promote nursing students harmonious development of physical and mental health.

\section{Reference}

[1] Lejie. Obstetrics and Gynecology [M]. sixth edition. Beijing: People's Medical Publishing House, 2004.9.

[2] Zhang Xingdong, horse volunteer, Li Xiaohong. Dysmenorrhea of female college students of psychosomatic therapy [J]. Maternal and child health care of China, 2007, 22 (35):5083.

[3] Liu Hua. Development of health education and health promotion [J]. China general practice medicine, 2001, 4 (10):757-758.

Author brief introduction: Cui Haina (06 December 1982), female, undergraduate, Shandong Dongying, Binzhou vocational college nursing college full-time teachers, research direction for basic nursing.

Tel: 18769649157

E-mail:cuihaina421@126.com 